\title{
MR-BASED ATTENUATION CORRECTION IN BRAIN PET/MR STUDIES: A SHORT REVIEW
}

\author{
Elisabetta Giovannini, Giampiero Giovacchini, Stefania Nicolosi, Mattia Riondato \\ and Andrea Ciarmiello* \\ Nuclear Medicine Department, S. Andrea Hospital, La Spezia, Italy
}

(History: received 15 March 2017; accepted 06 April 2017; published online 10 April 2017)

\begin{abstract}
In the previous few years we have witnessed to an increased diffusion of positron emission tomography/magnetic resonance (PET/MR) tomographs and equally an increasing number of clinical studies with these hybrid devices in both the neurological and psychiatric fields. Although PET/MR contains many features that facilitate its application in brain imaging, accurate quantification is still hindered by difficulties in developing MR-based attenuation correction methods. In this paper, we have reviewed the three main methods currently used for attenuation correction in PET/MR: namely segmentation-based methods including, atlas and template. In addition to procedures based on the combination of PET emission data and MR anatomical information (or reconstruction-based methods). Many research centers are actively working to refine available methods and substantial improvements are expected in future years. Clinical studies using PET/MR focused mainly on neurooncological and neurodegenerative disorders. Simultaneous PET/MR was shown to provide very promising scientific results and to be logistically more convenient for patients. More studies are expected in the near future, as the availability of PET/MR and the clinical use of new tracers for neurodegenerative disorders will further increase.
\end{abstract}

Keywords: PET/MR; attenuation correction; brain tumors; neurodegenerative disorders

\section{INTRODUCTION}

$\mathrm{T}$ lechnical developments in the last decade have permitted the construction of hybrid positron emission tomography/magnetic resonance (PET/MR) devices. In comparison to positron emission tomography/computed tomography (PET/CT) which is the current gold standard hybrid device used in routine clinical practice, PET/MR offers some advantages, including reduced ionizing radiation, improved soft-tissue contrast, the possibility to perform an MR-based motion correction and partial volume correction without an additional external acquisition and the acquisition of fused, simultaneous and

\section{A OPEN ACCESS PEER REVIEWED}

*Correspondence E-mail: andrea.ciarmiello@ as15.liguria.it

Citation: Ciarmiello A. MR-based attenuation correction in brain PET/MR studies: A short review. Journal of Diagnostic Imaging in Therapy. 2017; 4(1): 29-34.

http://dx.doi.org/10.17229/jdit.2017-0410-028

Copyright: (c) 2017 Ciarmiello A. This is an open-access article distributed under the terms of the Creative Commons Attribution License (CC By 4.0), which permits unrestricted use, distribution, and reproduction in any medium, provided the original author and source are cited. multiparametric images that provide morphological and functional information [1-3]. The first PET/MR devices were built [4-5] even before the introduction of PET/CT [6]. Despite more than two decades of extensive research on PET/MR, only a small number of centres are currently equipped with these devices [7]. Several technical difficulties make the combination of these two modalities challenging (see for review [3,7]) and PET/MR also presents some disadvantages.

From a logistical point of view, these disadvantages include high prices, the need for highly qualified interdisciplinary personnel for maintenance and possibly with the exception of only brain studies still limited clinical breakthroughs as compared to PET/CT [8]. The first integrated PET/MR imaging study of the human brain was published in 2008 [9] and in the last few years we have observed an increasing application of PET/MR in the neurological field [2]. In as far as brain imaging is concerned, the main disadvantage is represented by the difficulty of performing accurate attenuation correction, which is a prerequisite for quantification of PET data. In this article, we will concisely review the theoretical framework underlying the difficulties and also the possible solutions to address this problem. 


\section{ATTENUATION CORRECTION IN PET/MR: THE GENERAL PROBLEM}

Accurate quantification requires correction for attenuation of the $511 \mathrm{keV}$ gamma rays. This is important for routine clinical studies as well as for more sophisticated research protocols requiring, for example, parameter estimation [10]. Photon attenuation is related to electron density. CT-based attenuation correction has been implemented in current hybrid PET/CT scanners. This correction is linear to the electron density. However, the MR signal depends on proton density and tissue relaxation properties, so that there is no relationship between MR image intensity and photon attenuation [10-11].

Additional challenges are represented by the fact that standard MR sequences provide only a very low signal in the skeleton because of the low proton density and the very short T2 of bone $[3,11]$. Therefore, differentiation of bone and air through the MR signal obtained from standard sequences is not feasible. Since their attenuation coefficients are very different, alternative strategies are needed to be developed. Consequently, MR-based attenuation correction (MRAC) methods designed for cerebral studies must correct for bone attenuation [12-14]. For this purpose, ultrashort echo time (UTE) sequences as well as dual ultrashort echo (DUTE) were introduced; these sequences are able to visualize tissues with very short T2 relaxation time, such as the skeleton, with sufficient signalto-noise ratio [12,15-17].

All attenuating objects inside the field-of-view need to be corrected for. While this is true for any PET system, a particular problem of PET-MR hybrid scanners is represented by the fact that MR coils and other hardware which are positioned close to the patient - induce photon attenuation but no significant MR signal in traditional sequences and their interference also needs to be accurately corrected for $[3,5,9,16]$.

There are some general requirements of an "ideal" method of attenuation correction for PET/MR [3,18]. Probably, the most important requirement is represented by the robustness against intra- and inter-subject variability in all brain regions, which results from the combination of accuracy and reproducibility, in order to avoid errors in the attenuation map and in quantification. The accuracy of these methods is tested against the attenuation correction obtained with CT, which is considered as the gold standard. Many factors, including hardware and software, ultimately contribute to the final robustness of a method. Since functioning of MR and PET software is independent, ideally the attenuation correction method for PET/MR hybrids should be simultaneous to the acquisition of PET data and not increase the total acquisition time, i.e. it should be performed rapidly.

\section{METHODS FOR ATTENUATION CORRECTION FOR PET/MR}

Schematically, MRAC methods can be divided into three main types on the basis of the technique applied to create the attenuation map.

\section{1) Segmentation-based methods}

A first class is represented by segmentation-based methods. These methods perform a segmentation of the various brain tissues and assign to each tissue class a predefined uniform linear attenuation coefficient. Segmentation-based methods are most frequently used and implemented in most commercial PET/MR devices. The ability to identify the number and the type of tissue segments largely depends on the MR sequences used. Anatomical regions are identified and assigned to the corresponding segments on the basis of the intensity of the MR signal or on the basis of their anatomical location. Segmentation-based methods usually use T1 [19] or UTE $[16,20]$ images.

In brain imaging, separation into three classes is generally adopted, as it is assumed that the histogram of attenuation values has three dominant peaks: air, soft tissue, and bone. The main advantage of segmentation methods is that they have the potential to account for the physiological intersubject and age- or pathology related variability in brain anatomy. This occurs because the procedure works on a voxel-by-voxel basis, i.e. very subtle changes in anatomy can be accurately processed $[3,14,16]$.

Segmentation methods have the following main limits: 1) segmentation errors and consequently classification errors may occur; for example, fine structures such as nasal cavities and cerebrospinal fluid (CSF) are sometimes misclassified [7] and lack of identification of the air cavities may introduce overestimations in adjacent brain areas [16]. This may be due to errors occurring during the execution of the mathematical function underlying the segmentation process and errors are favoured by limits in the available MR sequences [17]). 2) Several components of PET/MR devices, such as the table and the radiofrequency coils, do not provide MR signal and attenuation correction: for these structures cannot be performed by MR segmentation. Ignoring the attenuation caused by the radiofrequency coil can introduce substantial underestimations in the cerebral PET values [16]. 3) Predefined $\mu$ linear attenuation coefficient values are subject-independent $[3,14,16]$.

\section{2) Methods based on atlas or template}

These methods work through a three-dimensional adaptation of a CT atlas or of an attenuation-map template in order to obtain the patient's attenuation map. Application of the same deformation (or registration) to the atlas CT image generates the desired pseudo-CT volume. A direct mapping from MR to CT intensities cannot be performed because of lack of linearity. Therefore, CT-maps indirectly derived from MR were named pseudo-CT [21].

Several variants of this approach were published using non-rigid registration of measured attenuation maps [18,2125] or of an attenuation map produced from a tissue atlas [26]. These methods may work well, especially for the distinction of brain parenchyma, but they are often less accurate in regions with high anatomical variability [7]. Moreover, because of the anatomic adaptation during the registration process, these methods produce patient specific attenuation correction maps [18]. 
The advantage of this method is that the template provides structural anatomical information, which is directly related to electron density. In particular, since morphological information of the bone is already included in the template, the need of UTE acquisitions could be eliminated. Different $\mu$-values for different anatomical regions can be incorporated in the model. A disadvantage is represented by the fact that templates are made by scanning normal subjects. Therefore, when used in patients, they can induce errors that are expected to a larger amount as much normal anatomy is disrupted by the disease [3]. Atlas-based methods use Dixon, T1-, and/or T2-weighted sequences [27-28].

Atlas-based methods and segmentation methods were recently combined in an attempt to improve the outcome of the attenuation correction with promising preliminary results [18].

\section{3) Methods based on the combination of PET emission data and MR anatomical information or reconstruction- based methods}

These methods are based on iterative algorithms, and therefore are also referred to as reconstruction-based [29]. The most frequently used reconstruction function is the Maximum Likelihood reconstruction of Attenuation and Activity (MLAA). Many iterative programs have the capability to incorporate a priori anatomical or functional information in the reconstruction process [30]. Thus, the substitution of CT anatomy with MR anatomy has been a natural extension of these algorithms for PET/MR tomographs [31]. Incorporation of the MR information in the reconstruction loop to obtain the attenuation sinogram is a great advantage. Currently, these methods are those that require more evaluation for PET/MR brain applications.

The current availability of powerful software makes these computations sometimes demanding, technically possible and mathematically stable [32]. These techniques also have some important limitations: 1) a critical amount of radioactivity must be present in the anatomical regions in order to calculate regional values of the attenuation coefficient. While this holds true for tracers like $\left[{ }^{18} \mathrm{~F}\right]-\mathrm{FDG}$, other tracers, such as radiolabelled choline or amino acid tracers, do not have sufficient uptake in the normal brain because their uptake is limited by the integrity of the blood brain barrier. 2) Attenuating objects outside the patient do not have emission and correction for such objects (e.g. coils) and is critical for PET/MR. 3) Scatter correction is more difficult and it can induce crosstalk between the estimation of attenuation and emission. Time-of-flight technology may improve the accuracy of the estimation of the attenuation map [32].

\section{APPLICATION OF MRAC METHODS IN THE CLINICAL STUDIES}

The introduction of hybrid PET/MR systems allows simultaneous multimodality image acquisition of high technical quality. This technique is well suited for the brain considering that $\mathrm{MR}$ represents the first-line diagnostic imaging modality for numerous indications. Avoiding the repositioning of the patient improves co-registration and localization of anatomic structures and lesions [2-3]. We will now briefly summarize the results of some clinical studies applied in neuro-oncological and dementia patients that have shown the increasing clinical impact of PET/MR.

\section{Brain Tumours}

MR is firmly established as a diagnostic and assessment method of choice for brain tumour patients and has found increasing use as a cancer imaging biomarker [33-35].

Several quantitative MR methods (e.g., dynamic contrast-enhanced MR, dynamic susceptibility contrast MR, MR spectroscopy, and diffusion MR) have been used to improve cancer imaging. However, these MR techniques also have limitations, such as limited specificity. PET tracers for studying amino acid transport (e.g., $\left(\left[{ }^{11} \mathrm{C}\right]-\right.$ methionine and $\left[{ }^{18} \mathrm{~F}\right]$-fluoroethyltyrosine, FET), cellular proliferation (e.g., ([ $\left.{ }^{18} \mathrm{~F}\right]$-fluorothymidine), and tissue hypoxia (e.g., $\left[{ }^{18} \mathrm{~F}\right]$-fluoromisonidazole) have been demonstrated to have the potential to offset some of the existing limitations of MR for brain tumour diagnosis [3336].

In neuro-oncology the better characterization of various tissue types by combined metabolic and morphological imaging is of great importance in the differential diagnosis of brain tumours, for grading, for the assessment of progression and the distinction between necrosis and recurrence; PET/MR also helps in the selection of the most promising place for biopsies and in the evaluation of treatment effects and provides better results than either technique alone [37].

Henriksen et al. investigated the feasibility of simultaneous structural MR, blood volume (BV) derived from $M R$ and FET-PET of gliomas using an integrated PET/MR scanner. They also evaluated the spatial and quantitative agreement in tumour imaging between blood volume MR and FET PET. A total of 32 glioma patients underwent a simultaneous FET PET/MR acquisition. Maximal relative tumour FET uptake as tumour-tobackground ratio (TBRmax), relative BVmax (rBVmax), and Dice coefficients were calculated to assess the quantitative and spatial congruence in the tumour volumes determined by FET PET, BV MR and contrast-enhanced MR. FET volume and TBRmax were higher in BV-positive than in BV-negative scans, and both BV and rBVmax were higher in FET-positive than in FET-negative scans.

TBRmax and rBVmax were positively correlated. FET and BV positivity were in agreement in 26 of the $32(81 \%)$ patients and in 42 of $63(67 \%)$ lesions and spatial congruence in tumour volumes determined by MR, as assessed by the Dice coefficients and PET was generally poor. This study demonstrated that, although tumour volumes determined by $\mathrm{BV} \mathrm{MR}$ and FET PET were quantitatively correlated, their spatial congruence in a mixed population of treated glioma patients was generally poor and the modalities provided similar, but not identical, information in this population of patients [38].

The potential role of hybrid gadolinium $(\mathrm{Gd})$-enhanced FET-PET/MR in distinguishing brain tumour recurrence 
from radiation necrosis was investigated by Jena et al [39]. They analysed twenty-six patients with single or multiple contrast-enhancing brain lesions on MR after surgery and radiation therapy. Patients underwent simultaneous PET/MR and TBRmax, TBRmean, choline-to-creatine $(\mathrm{Cho} / \mathrm{Cr})$ ratios as well as $\mathrm{rCBVmean}$ and mean apparent diffusion coefficient (ADCmean) were determined.

Individually, TBRmax, TBRmean, ADCmean, and $\mathrm{Cho} / \mathrm{Cr}$ ratios as well as normalized $\mathrm{rCBVmean}$ provided reliable differentiation between radiation necrosis, with an accuracy of $93.8 \%$ for TBRmax, $87.5 \%$ for TBRmean, $81.3 \%$ for ADCmean, $96.9 \%$ for $\mathrm{Cho} / \mathrm{Cr}$ ratio, and $90.6 \%$ for normalized rCBVmean. The accuracy of both normalized rCBVmean and ADCmean was improved in combination with $\mathrm{TBRmax}$ or $\mathrm{Cho} / \mathrm{Cr}$ ratio. However, TBRmax (or TBRmean) with $\mathrm{Cho/Cr}$ ratio yielded the highest accuracy, approaching up to $97 \%$. Their findings suggested that FET uptake with $\mathrm{Cho} / \mathrm{Cr}$ ratio and normalized rCBVmean could be most useful in distinguishing primary glioma recurrence from radiation necrosis [39].

A very important advantage of PET/MR is also the reduction of radiation exposure taking place in paediatric patients. $\left[{ }^{18} \mathrm{~F}\right]$-fluorocholine PET/MR scans were performed in 12 patients with proven astrocytic tumours [40]. Eight patients simultaneously underwent $\left[{ }^{18} \mathrm{~F}\right]$-fluorocholine PET/MR follow-up scans after treatment. At baseline, the areas of $\left[{ }^{18} \mathrm{~F}\right]$-fluorocholine uptake matched areas of contrast enhancement and restricted diffusion. There was a negative correlation between SUVmax and ADCmean and a positive correlation between SUVmax and tumour size. There was concordance between reduction in tumour size and reductions in SUVmax and SUVmean in four children, in three of whom ADCmean values were increased. In two patients, tumour size remained stable whereas SUVmax and SUVmean values were increased with reduction of the ADCmean values. Additionally, in two children, MR showed an increase both in tumour size and SUVmax but a reduction in ADC values [40].

\section{Neurodegenerative disorders}

Neurodegenerative diseases include dementias, parkinsonian syndromes, corea and amyotrophic lateral sclerosis. In this group of disorders MR is frequently used as the initial examination in clinical routine to identify specific atrophy patterns and to exclude other pathologies. Similarly, brain PET has been used over many years to support the clinical diagnosis of neurodegenerative diseases [41]. The main advantage of PET with $\left[{ }^{18} \mathrm{~F}\right]-\mathrm{FDG}$ is its high sensitivity to detect pathologies at a molecular level, which can offer more sensitive or even earlier diagnoses, because these diseases start with biochemical processes that only lead to morphologic changes visible on MR after a certain time period [42]. In addition, new more specific PET tracers such as tracers that bind $\beta$-amyloid plaques, tau, $\alpha$ synuclein aggregates or tracing dopaminergic pathways integrity, have the potential to increase the diagnostic abilities of combined PET/MR technology providing the possibility to improve the early and differential diagnosis of many neurodegenerative diseases $[41,43]$.

In order to evaluate the qualitative performance of PET/MR in clinical neuroimaging, Hitz et al. compared results obtained with integrated $\mathrm{PET} / \mathrm{MR}$ with conventional PET/CT in thirteen patients for assessment of cognitive impairment [44]. Attenuation and scatter correction were performed using low-dose CT for the PET/CT and segmented Dixon MR imaging data for the PET/MR. Comparison between PET/MR and PET/CT were assessed by evaluation of region-of-interest (ROI). Individual PET/MR and PET/CT datasets were compared versus a predefined independent control population [44]. Despite AC, lower measured PET signal values were found throughout the brain cortex in ROI-based quantification of the PET signal for PET/MR as compared with PET/CT. On the contrary, significantly higher relative signals in the subcortical and basal regions of the brain than the corresponding PET/CT images of the AC data.

Further insights into the development of cognitive disturbances have been obtained through PET studies of deposition of amyloid, tau, or other abnormal proteins of degenerative disorders. For example, $\mathrm{Su}$ investigated the impact of using a standard MR-based attenuation correction technique on the clinical and research utility of a PET/MR hybrid scanner for amyloid imaging [45]. $\left[{ }^{18} \mathrm{~F}\right]$-Florbetapir was used as the radiopharmaceutical to detect beta-amyloid deposits. Forty subjects were enrolled in the study. The scans were obtained on a hybrid scanner with simultaneous PET/MR acquisition. In MR-based attenuation correction PET measurements were underestimated in comparison to the gold standard in the majority of the cerebral areas and they were slightly overestimated in subcortical structures. Moreover, there was an overestimation of SUVRs using the cerebellum as the reference region. The quantitative differences, however, did not affect visual assessment as either positive or negative [45].

\section{CONCLUSION}

Technological developments in the last few years have contributed to an increased installation of PET/MR tomographs in selected centres and to an increasing number of neurological studies with these hybrid devices. While PET/MR has many features that facilitate clinical use in the neurological field, the main limitation lies in the difficulty of performing accurate quantification, which is often desired in brain PET imaging. We have briefly reviewed the three main methods currently used for attenuation correction in PET/MR tomographs. Segmentation-based methods and atlas- or template-based methods are the ones most commonly used today, whilst reconstruction-based methods still require some larger validation refinement. Quantification of brain PET values is very sensitive to the accurate segmentation of bone and generally to the precise quantification of bone attenuation. Many research groups are actively working to refine available methods and significant improvements have been completed in the previous few years. 
Clinical studies using PET/MR are increasing as the technique finds increasing clinical acceptance. An immediate advantage for the patient requiring both a morphological and a functional study is that both examinations can be performed at the same time in the same centre. Results of the limited studies available show that the use of PET/MR provides results overall comparable to those obtained by PET/CT and MR acquired independently. More studies are expected in the near future, as the availability of PET/MR and the clinical use of new tracers for neurodegenerative disorders will increase.

\section{CONFLICTS OF INTEREST}

The authors report no conflicts of interest.

\section{REFERENCES}

Key References:10, 12, 18, 37, 40

[1] Catana C, Benner T, van der Kouwe A, Byars L, Hamm M, Chonde DB, et al. MRI-assisted PET motion correction for neurologic studies in an integrated MR-PET scanner. J. Nucl. Med. 2011; 52: 154-161. CrossRef PubMed

[2] Catana C, Drzezga A, Heiss WD, Rosen BR. PET/MRI for neurologic applications. J. Nucl. Med. 2012; 53: 1916-1925. CrossRef PubMed

[3] Vandenberghe S, Marsden PK. PET-MRI: a review of challenges and solutions in the development of integrated multimodality imaging. Phys. Med. Biol. 2015; 60: R115-154. CrossRef PubMed

[4] Hammer BE, Christensen NL, Heil BG. Use of a magnetic field to increase the spatial resolution of positron emission tomography. Med. Phys. 1994; 21: 1917-1920. CrossRef PubMed

[5] Shao Y, Cherry SR, Farahani K, Meadors K, Siegel S, Silverman RW, et al. Simultaneous PET and MR imaging. Phys. Med. Biol. 1997; 42: 1965-1970. CrossRef PubMed

[6] Beyer T, Townsend DW, Brun T, Kinahan PE, Charron M, Roddy R, et al. A combined PET/CT scanner for clinical oncology. J. Nucl. Med. 2000; 41: 1369-1379.

CrossRef PubMed

[7] Disselhorst JA, Bezrukov I, Kolb A, Parl C, Pichler BJ. Principles of PET/MR Imaging. J. Nucl. Med. 2014; 55: 2S-10S. CrossRef PubMed

[8] Wieser G, Mansi R, Grosu AL, Schultze-Seemann W, Dumont-Walter RA, Meyer PT, et al. Positron emission tomography (PET) imaging of prostate cancer with a gastrin releasing peptide receptor antagonist-from mice to men. Theranostics. 2014; 4: 412-419.

CrossRef PubMed

[9] Schlemmer HP, Pichler BJ, Schmand M, Burbar Z, Michel C, Ladebeck R, et al. Simultaneous MR/PET imaging of the human brain: feasibility study. Radiology. 2008; 248: 1028-1035. CrossRef PubMed

[10]Beyer T, Weigert M, Quick HH, Pietrzyk U, Vogt F, Palm C, et al. MR-based attenuation correction for torso-PET/MR imaging: pitfalls in mapping MR to CT data. Eur. J. Nucl. Med. Mol. Imaging. 2008; 35: 1142-1146. CrossRef PubMed

[11] Stanisz GJ, Odrobina EE, Pun J, Escaravage M, Graham SJ, Bronskill MJ, et al. T1, T2 relaxation and magnetization transfer in tissue at 3T. Magn. Reson. Med. 2005; 54: 507-512. CrossRef PubMed

[12]Berker Y, Franke J, Salomon A, Palmowski M, Donker HC, Temur Y, et al. MRI-based attenuation correction for hybrid PET/MRI systems: a 4-class tissue segmentation technique using a combined ultrashortecho-time/Dixon MRI sequence. J. Nucl. Med. 2012; 53: 796-804. CrossRef PubMed
[13] Andersen FL, Ladefoged CN, Beyer T, Keller SH, Hansen AE, Hojgaard L, et al. Combined PET/MR imaging in neurology: MRbased attenuation correction implies a strong spatial bias when ignoring bone. NeuroImage. 2014; 84: 206-216. CrossRef PubMed

[14]Bezrukov I, Mantlik F, Schmidt H, Scholkopf B, Pichler BJ. MRBased PET attenuation correction for PET/MR imaging. Semin. Nucl. Med. 2013; 43: 45-59.

CrossRef PubMed

[15] Martinez-Moller A, Souvatzoglou M, Delso G, Bundschuh RA, Chefd'hotel C, Ziegler SI, et al. Tissue classification as a potential approach for attenuation correction in whole-body PET/MRI: evaluation with PET/CT data. J. Nucl. Med. 2009; 50: 520-526. CrossRef PubMed

[16] Catana C, van der Kouwe A, Benner T, Michel CJ, Hamm M, Fenchel $\mathrm{M}$, et al. Toward implementing an MRI-based PET attenuationcorrection method for neurologic studies on the MR-PET brain prototype. J. Nucl. Med. 2010; 51: 1431-1438. CrossRef PubMed

[17] Keereman V, Fierens Y, Broux T, De Deene Y, Lonneux M, Vandenberghe S. MRI-based attenuation correction for PET/MRI using ultrashort echo time sequences. J. Nucl. Med. 2010; 51: 812818.

CrossRef PubMed

[18] Sekine T, Buck A, Delso G, Ter Voert EE, Huellner M, Veit-Haibach $\mathrm{P}$, et al. Evaluation of Atlas-Based Attenuation Correction for Integrated PET/MR in Human Brain: Application of a Head Atlas and Comparison to True CT-Based Attenuation Correction. J. Nucl. Med. 2016; 57: 215-220. CrossRef PubMed

[19]Zaidi H, Montandon ML, Slosman DO. Magnetic resonance imagingguided attenuation and scatter corrections in three-dimensional brain positron emission tomography. Med. Phys. 2003; 30: 937-948. CrossRef PubMed

[20] Cabello J, Lukas M, Forster S, Pyka T, Nekolla SG, Ziegler SI. MRbased attenuation correction using ultrashort-echo-time pulse sequences in dementia patients. J. Nucl. Med. 2015; 56: 423-429. CrossRef PubMed

[21] Hofmann M, Steinke F, Scheel V, Charpiat G, Farquhar J, Aschoff P, et al. MRI-based attenuation correction for PET/MRI: a novel approach combining pattern recognition and atlas registration. J. Nucl. Med. 2008; 49: 1875-1883. CrossRef PubMed

[22] Montandon ML, Zaidi H. Atlas-guided non-uniform attenuation correction in cerebral 3D PET imaging. NeuroImage. 2005; 25: 278286 CrossRef PubMed

[23] Montandon ML, Zaidi H. Quantitative analysis of template-based attenuation compensation in 3D brain PET. Comput. Med. Imaging Graph. 2007; 31: 28-38. CrossRef PubMed

[24] Kops ER, Herzog H. Alternative methods for attenuation correction for PET images in MR-PET scanners. IEEE Nucl Sci Symp Conf Rec. 2007; 6: 4327-4330. CrossRef

[25] Schreibmann E, Nye JA, Schuster DM, Martin DR, Votaw J, Fox T. MR-based attenuation correction for hybrid PET-MR brain imaging systems using deformable image registration. Med. Phys. 2010; 37: 2101-2109. CrossRef PubMed

[26]Zaidi H, El Naqa I. PET-guided delineation of radiation therapy treatment volumes: a survey of image segmentation techniques. Eur. J. Nucl. Med. Mol. Imaging. 2010; 37: 2165-2187. CrossRef PubMed

[27] Andreasen D, Van Leemput K, Hansen RH, Andersen JA, Edmund JM. Patch-based generation of a pseudo CT from conventional MRI sequences for MRI-only radiotherapy of the brain. Med. Phys. 2015; 42: 1596-1605. CrossRef PubMed 
[28] Torrado-Carvajal A, Herraiz JL, Alcain E, Montemayor AS, GarciaCanamaque L, Hernandez-Tamames JA, et al. Fast Patch-Based Pseudo-CT Synthesis from T1-Weighted MR Images for PET/MR Attenuation Correction in Brain Studies. J. Nucl. Med. 2016; 57: 136143. CrossRef PubMed

[29] Ladefoged CN, Law I, Anazodo U, St Lawrence K, Izquierdo-Garcia $\mathrm{D}$, Catana C, et al. A multi-centre evaluation of eleven clinically feasible brain PET/MRI attenuation correction techniques using a large cohort of patients. NeuroImage. 2016; 147: 346-359. CrossRef PubMed

[30]Delso G, Martinez-Moller A, Bundschuh RA, Nekolla SG, Ziegler SI. The effect of limited MR field of view in MR/PET attenuation correction. Med. Phys. 2010; 37: 2804-2812. CrossRef PubMed

[31] Nuyts J, Dupont P, Stroobants S, Benninck R, Mortelmans L, Suetens P. Simultaneous maximum a posteriori reconstruction of attenuation and activity distributions from emission sinograms. IEEE Trans. Med. Imaging. 1999; 18: 393-403.

CrossRef PubMed

[32] Rezaei A, Defrise M, Bal G, Michel C, Conti M, Watson C, et al. Simultaneous reconstruction of activity and attenuation in time-offlight PET. IEEE Trans. Med. Imaging. 2012; 31: 2224-2233. CrossRef PubMed

[33] Sorensen AG. Magnetic resonance as a cancer imaging biomarker. $J$. Clin. Oncol. 2006; 24: 3274-3281.

CrossRef PubMed

[34]Hamstra DA, Rehemtulla A, Ross BD. Diffusion magnetic resonance imaging: a biomarker for treatment response in oncology. J. Clin. Oncol. 2007; 25: 4104-4109.

CrossRef PubMed

[35] Aronen HJ, Gazit IE, Louis DN, Buchbinder BR, Pardo FS, Weisskoff RM, et al. Cerebral blood volume maps of gliomas: comparison with tumor grade and histologic findings. Radiology. 1994; 191: 41-51. CrossRef PubMed

[36] Hylton N. Dynamic contrast-enhanced magnetic resonance imaging as an imaging biomarker. J. Clin. Oncol. 2006; 24: 3293-3298. CrossRef PubMed

[37] Neuner I, Kaffanke JB, Langen KJ, Kops ER, Tellmann L, Stoffels G, et al. Multimodal imaging utilising integrated MR-PET for human brain tumour assessment. European radiology. 2012; 22: 2568-2580. CrossRef PubMed
[38] Henriksen OM, Larsen VA, Muhic A, Hansen AE, Larsson HB, Poulsen HS, et al. Simultaneous evaluation of brain tumour metabolism, structure and blood volume using [(18)F]fluoroethyltyrosine (FET) PET/MRI: feasibility, agreement and initial experience. Eur. J. Nucl. Med. Mol. Imaging. 2016; 43: 103-112. CrossRef PubMed

[39] Jena A, Taneja S, Gambhir A, Mishra AK, D'Souza M M, Verma SM, et al. Glioma Recurrence Versus Radiation Necrosis: Single-Session Multiparametric Approach Using Simultaneous O-(2-18FFluoroethyl)-L-Tyrosine PET/MRI. Clin. Nucl. Med. 2016; 41: e228236. CrossRef PubMed

[40]Fraioli F, Shankar A, Hargrave D, Hyare H, Gaze MN, Groves AM, et al. 18F-fluoroethylcholine (18F-Cho) PET/MRI functional parameters in pediatric astrocytic brain tumors. Clin. Nucl. Med. 2015; 40: e4045. CrossRef PubMed

[41]Barthel H, Schroeter ML, Hoffmann KT, Sabri O. PET/MR in dementia and other neurodegenerative diseases. Semin. Nucl. Med. 2015; 45: 224-233. CrossRef PubMed

[42]Bohnen NI, Djang DS, Herholz K, Anzai Y, Minoshima S. Effectiveness and safety of 18F-FDG PET in the evaluation of dementia: a review of the recent literature. J. Nucl. Med. 2012; 53: 5971. CrossRef PubMed

[43] Klunk WE, Engler H, Nordberg A, Wang Y, Blomqvist G, Holt DP, et al. Imaging brain amyloid in Alzheimer's disease with Pittsburgh Compound-B. Annals of neurology. 2004; 55: 306-319. CrossRef PubMed

[44]Hitz S, Habekost C, Furst S, Delso G, Forster S, Ziegler S, et al. Systematic Comparison of the Performance of Integrated Whole-Body PET/MR Imaging to Conventional PET/CT for (1)(8)F-FDG Brain Imaging in Patients Examined for Suspected Dementia. J. Nucl. Med. 2014; 55: 923-931. CrossRef PubMed

[45] Su Y, Rubin BB, McConathy J, Laforest R, Qi J, Sharma A, et al. Impact of MR-Based Attenuation Correction on Neurologic PET Studies. J. Nucl. Med. 2016; 57: 913-917.

CrossRef PubMed 